\title{
Portal lymphadenopathy associated with lipofuscin in chronic cholestatic liver disease
}

\author{
S G HÜBSCHER, R F HARRISON \\ From the Department of Pathology, The Medical School, University of Birmingham, Birmingham
}

SUMMARY To determine whether portal lymphadenopathy in primary biliary cirrhosis is caused by deposition of lipofuscin pigment in sinus histiocytes and to compare primary biliary cirrhosis with other liver diseases a retrospective study on a consecutive series of 169 livers obtained at transplantation was carried out. There were grouped into eight diagnostic categories: primary biliary cirrhosis $(n=51)$, primary sclerosing cholangitis $(n=10)$, extrahepatic biliary atresia $(n=6)$, chronic rejection $(n=9)$, cirrhosis (other causes) $(n=38)$, primary liver neoplasia $(n=21)$, acute liver disease $(n=20)$, and retransplantation (other) $(n=14)$. Lymph nodes were present in 66 specimens. Fifty of these contained granules of lipofuscin pigment. The highest incidence of lymph node enlargement and the largest amounts of pigment were present in cases of primary biliary cirrhosis. A similar pattern of lymph node enlargement was also commonly observed in other chronic cholestatic conditions (primary sclerosing cholangitis, biliary atresia, chronic rejection). Much less pigment was seen in nodes draining livers with non-cholestatic cirrhosis or primary tumours. Nodes were not found in acute liver disease.

It is concluded that portal lymphadenopathy associated with lipofuscin is a common finding in various chronic cholestatic liver diseases. The pathogenesis of this lesion is uncertain. Most cases arę asymptomatic with enlarged nodes which may be detected only at laperotomy or necropsy and mays be wrongly attributed to neoplastic disease. Diagnostically, the finding of large amounts of lipofuscin in enlarged portal lymph nodes is a good indicator of underlying chronic cholestatic liver disease.

Enlarged, pigmented portal lymph nodes are often seen in end-stage primary biliary cirrhosis. The pigment, initially thought to be bile, ${ }^{1}$ has recently been shown to have the staining characteristics of lipofuscin in two cases reported by Benbow and McMahon. ${ }^{2}$ To investigate this phenomenon further and to compare primary biliary cirrhosis with other liver diseases we carried out a retrospective histological and histochemical study on lymph nodes attached to livers obtained at liver transplantation.

\section{Material and methods}

Histological material was examined retrospectively from the first 169 liver transplants carried out in Birmingham between January 1982 and September 1987. All blocks which contained lymph nodes (excluding those with metastatic tumour deposits) were further examined by histological and histo-

Accepted for publication 25 May 1989 chemical techniques. An estimate of lymph node size was obtained for each case by measuring the maximum dimension of the largest node present in histological sections.

Each case was placed into one of eight diagnostic categories on the basis of characteristic clinical, biochemical, immunological, radiological and histological features. The eight categories were: primary biliary cirrhosis $(n=51)$, primary sclerosing cholangitis $(n=10)$, extrahepatic biliary atresia $(n=6)$, chronic liver allograft rejection $(n=9)$, cirrhosis (other causes) $(n=38)$, acute liver disease $(n=20)$, primary liver tumour $(n=21)$, and retransplantation (other causes) $(n=14)$.

Further details of individual cases in each category are as follows:

Chronic rejection was characterised by loss of bile ducts and intimal foam cell lesions in large and medium sized arteries. ${ }^{3-5}$

Cirrhosis (other) comprised nine cases of $\alpha-1$ antitrypsin deficiency, eight of autoimmune chronic active hepatitis, four of chronic Budd-Chiari 
syndrome, three of chronic hepatitis B infection, two of alcoholic liver disease and one each of post-necrotic scarring, cystic fibrosis, and chronic erythropoietic protoporphyria. Nine cases were classified as cryptogenic.

Primary liver tumours comprised 13 cases of hepatocellular carcinoma (nine arising in cirrhotic livers), five of fibrolamellar carcinoma, and one each of cholangiocarcinoma, epithelioid haemangioendothelioma, and angiosarcoma. Lymph nodes containing metastatic tumour deposits were excluded from this study.

Acute liver disease comprised 14 cases of fulminant non-A, non-B hepatitis, ${ }^{6}$ four of acute Budd-Chiari syndrome, and one of fulminant hepatitis B infection. In the remaining case an idiosyncratic reaction to paint spray was implicated.

Indications for transplantation in the other 14 cases retransplanted were ischaemia/infarction $(n=8)$ and acute massive haemorrhagic necrosis $(n=5)^{7}$. The remaining case showed severe cholestasis with no specific clues to aetiology.

Lymph nodes from each case were stained with the following: (i) Long Ziehl-Neelsen (long $\mathrm{ZN}$ ) for lipofuscin; (ii) periodic acid Schiff after diastase treatment (PAS-D) for ceroid; (iii) Perls's reaction for haemosiderin; (iv) Shikata's orcein stain for copperassociated protein; and (v) haematoxylin van Gieson (HVG) for bile. The amount of pigment shown by these methods was graded semiquantitatively on a scale of $0-3+$.

\section{Results}

INCIDENCE AND DEGREE OF NODE ENLARGEMENT Lymph nodes were present in 66 of the 169 specimens examined. Table 1 summarises the number of cases with nodes, the total number of nodes present, and the maximum node dimension in each diagnostic category.

HISTOLOGICAL FINDINGS

All of the nodes examined showed histological abnormalities, although these were often minor. Two main patterns were identified: Fifty of the 66 cases with lymph nodes showed sinus histiocytosis with granular deposits of brown pigment in sinus macrophages (fig la). The shape and size of pigment granules varied. Most were round to-oval in shape and measured $1-5 \mu \mathrm{m}$ in diameter. The amount of pigment deposited in sinus histiocytes seemed to correlate with the degree of sinus histiocytosis present. Twenty four cases in this group also showed varying degrees of follicular hyperplasia. This was generally mild and did not contribute significantly to lymph node enlargement. The remaining 16 cases showed a mixed pattern of follicular hyperplasia and simple sinus histiocytosis without pigment deposition.

Other abnormalities noted in one or both of the above groups were as follows: Deposits of granular eosinophilic material in paracortical/mantle zone histiocytes ( 29 cases) were present in small amounts, were often difficult to see on haematoxylin and eosin sections, and were more clearly shown with PASdiastase staining (see below). They occurred with equal incidence in the two groups described above -22 of 50 cases with pigment $(44 \%)$, seven of 16 cases without pigment (44\%).

Lipogranulomas in medullary sinuses (28 cases) were also present in both groups described above-18 of 50 cases with pigment $(36 \%)$ and 10 of 16 cases without pigment $(62 \%)$. Epithelioid granulomas (five cases) were all seen in cases of primary biliary cirrhosis and also had pigment deposits in sinus histiocytes. There were two cases of anthracotic deposits in sinus macrophages.

\section{HISTOCHEMICAL FINDINGS \\ Lipofuscin}

Pigment in sinus histiocytes gave a strongly positive reaction for lipofuscin with the long $\mathrm{ZN}$ stain (fig lb). This material also stained positively to a variable degree with PAS-diastase. The amount of pigment shown by long ZN staining is shown in table 2 . Overall, much larger amounts of pigment were present in nodes from livers with chronic cholestasis (primary

Table 1 Incidence and degree of portal lymph node enlargement in 169 liver transplants

\begin{tabular}{|c|c|c|c|c|}
\hline Histological diagnosis & $\begin{array}{l}\text { No of } \\
\text { cases }\end{array}$ & $\begin{array}{l}\text { No (\%) with } \\
\text { nodes }\end{array}$ & $\begin{array}{l}\text { No of } \\
\text { nodes }\end{array}$ & $\begin{array}{l}\text { Median max } \\
\text { node dimension }(\mathrm{mm})\end{array}$ \\
\hline $\begin{array}{l}\text { Primary biliary cirrhosis } \\
\text { Primary sclerosing cholangitis } \\
\text { Biliary atresia } \\
\text { Chronic rejection } \\
\text { Cirrhosis (other) } \\
\text { Primary tumour } \\
\text { Acute liver disease } \\
\text { Retransplantation (other) }\end{array}$ & $\begin{array}{r}51 \\
10 \\
6 \\
9 \\
38 \\
21 \\
20 \\
14\end{array}$ & $\begin{array}{r}34(67) \\
5(50) \\
1(17) \\
5(56) \\
8(21) \\
13(62) \\
0(0) \\
0(0)\end{array}$ & $\begin{array}{r}75 \\
18 \\
3 \\
9 \\
18 \\
46 \\
- \\
-\end{array}$ & $\begin{array}{r}10 \\
14 \\
14 \\
8 \\
9 \\
12 \\
- \\
-\end{array}$ \\
\hline Total & 169 & $66(39)$ & 169 & 11 \\
\hline
\end{tabular}



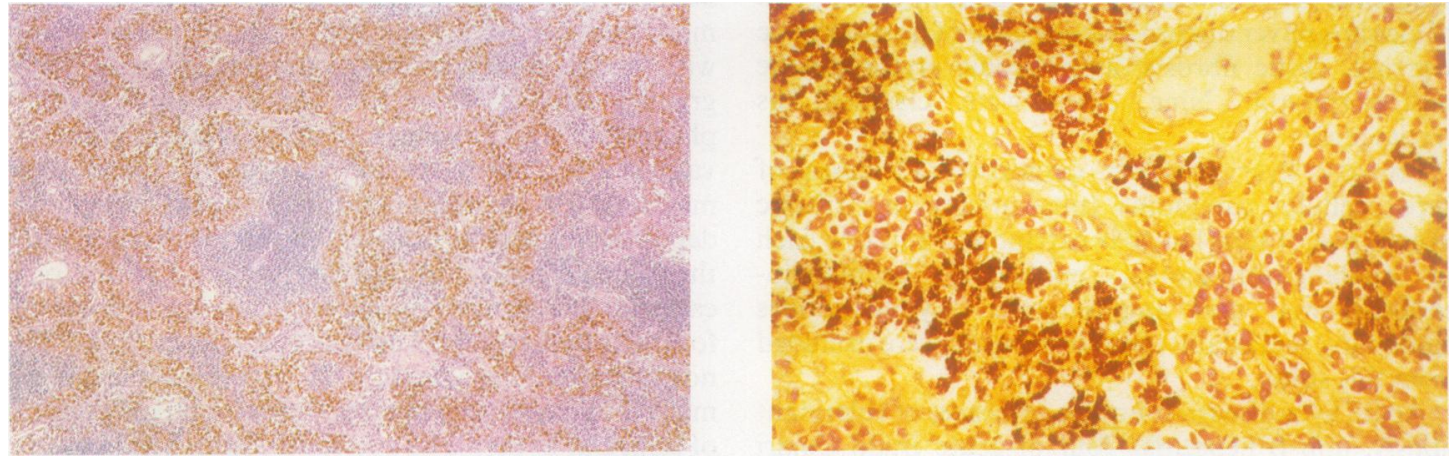

Fig 1 (a) Portal lymph node showing expansion of medullary sinuses associated with deposits of brown pigment (from a case of primary biliary cirrhosis). (Haematoxylin and eosin.) (b) Sinus histiocytes contain granules of varying sizes which stain as lipofuscin (same node as illustrated in fig la). (Long ZN.)

Table 2 Amount of lipofuscin in 66 cases with enlarged portal lymph nodes

\begin{tabular}{|c|c|c|c|c|c|}
\hline \multirow[b]{2}{*}{ Primary disease } & \multicolumn{4}{|c|}{ Amount of lipofuscin present } & \multirow{2}{*}{$\begin{array}{l}\text { Total No of } \\
\text { cases with nodes }\end{array}$} \\
\hline & 0 & $1+$ & $2+$ & $3+$ & \\
\hline $\begin{array}{l}\text { Primary biliary cirrhosis } \\
\text { Primary sclerosing cholangitis } \\
\text { Biliary atresia } \\
\text { Chronic rejection } \\
\text { Cirrhosis (other) } \\
\text { Primary tumour }\end{array}$ & $\begin{array}{r}0 \\
1 \\
0 \\
1 \\
4 \\
10\end{array}$ & $\begin{array}{l}2 \\
0 \\
0 \\
1 \\
3 \\
2\end{array}$ & $\begin{array}{r}12 \\
0 \\
0 \\
2 \\
0 \\
1\end{array}$ & $\begin{array}{r}20 \\
4 \\
1 \\
1 \\
1 \\
0\end{array}$ & $\begin{array}{r}34 \\
5 \\
1 \\
5 \\
8 \\
13\end{array}$ \\
\hline Total & 16 & 8 & 15 & 27 & 66 \\
\hline
\end{tabular}

biliary cirrhosis, primary sclerosing cholangitis, biliary atresia, chronic rejection) than in nodes from non-cholestatic livers (cirrhosis-other causes, primary hepatic neoplasia).

\section{Ceroid pigment}

Fifty four cases had deposits of granular material in paracortical and mantle zone histiocytes. This stained as ceroid with PAS-diastase (fig 2), was negative with

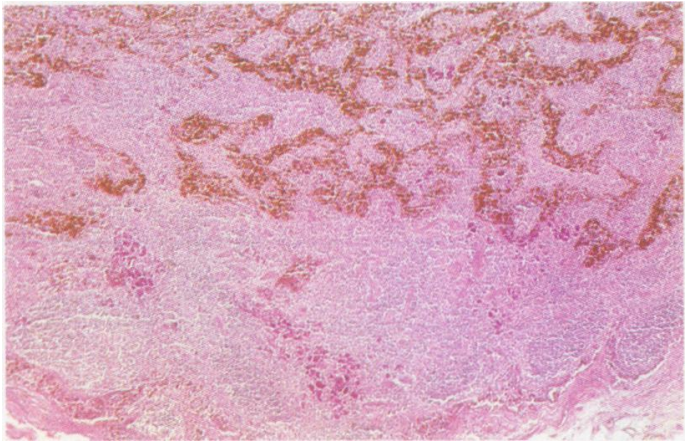

Fig 2 A separate population of ceroid-laden paracortical histiocytes is identified by PAS-diastase staining. Brown pigment in sinus macrophages is not stained by this method (from a case of primary biliary cirrhosis). (PAS-diastase.) long $\mathrm{ZN}$, and corresponded to the eosinophilic granules noted in haematoxylin and eosin sections (see above). Ceroid pigment was present in $\mathbf{4 6}$ of 50 cases with lipofuscin pigment and eight of 16 cases without lipofuscin pigment. Overall, ceroid pigment was present in considerably smaller quantities than lipofuscin, and the presence of ceroid laden paracortical/mantle zone macrophages did not contribute greatly to lymph node enlargement.

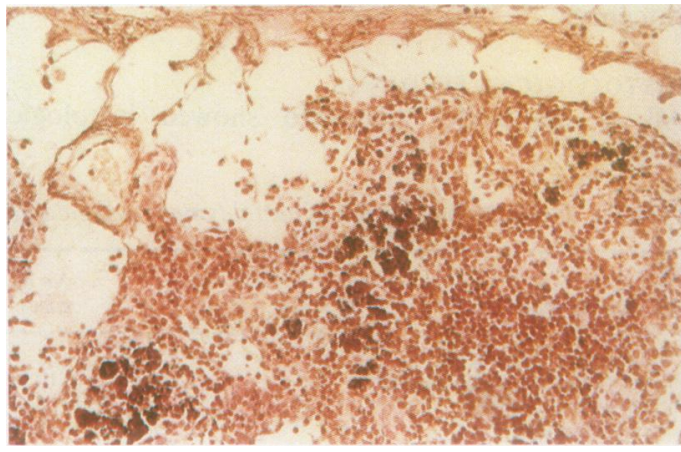

Fig 3 Paracortical histiocytes contain black granules with the staining properties of copper-associated protein (from a case of primary sclerosing cholangitis). (Orcein.) 


\section{Haemosiderin}

Perls's stain showed haemosiderin deposits in 12 cases. These were present in small quantities and were randomly distributed in the cortex and medulla.

\section{Copper-associated protein}

Granular deposits were demonstrable by orcein staining in $\mathbf{4 4}$ cases. These gave a variable staining reaction. Most granules were pinkish-brown in colour, present in paracortical and mantle zone histiocytes, and corresponded to ceroid pigment shown by PASdiastase staining. In 15 cases there were black granules of varying size and shape, corresponding to the staining reaction seen with copper-associated protein (fig 3). These again were mainly present in paracortical/mantle zone histiocytes, although scanty deposits were occasionally seen in medullary sinuses. Of the 15 cases with black granular deposits, there were 13 of primary biliary cirrhosis, one of primary sclerosing cholangitis, and one of chronic rejection. All 15 cases were associated with moderate to heavy lipofuscin deposits in sinus histiocytes.

\section{Bile}

The Van Gieson stain was negative for bile in all cases.

\section{Discussion}

Our study confirms that portal lymphadenopathy is common in long standing primary biliary cirrhosis, ${ }^{1}$ over two thirds of livers obtained at transplantation for primary biliary cirrhosis having readily detectable lymph nodes in the porta hepatis. All of the lymph nodes examined from cases of primary biliary cirrhosis had deposits of lipofuscin in sinus histiocytes. In most cases large amounts of pigment were present and were associated with proliferation of histiocytes and expansion of medullary sinuses. These observations support the recent suggestion that lipofuscin deposition is the main factor causing lymph node enlargement in primary biliary cirrhosis. ${ }^{2}$

Very little information exists concerning the normal size of lymph nodes in the porta hepatis and some of the nodes we studied may fall into a "normal range" as far as size is concerned. The fact that all of the nodes examined contained lipofuscin granules, however, suggests that lymph nodes in primary biliary cirrhosis are abnormal, irrespective of size. Furthermore, measurements of lymph nodes were carried out on fixed, processed tissue which may considerably underestimate their true size.

Although portal lymphadenopathy associated with lipofuscin is a common finding in primary biliary cirrhosis, it is clearly not confined to this condition. We have found a similar pattern of lymph node enlargement in other chronic liver diseases-notably, those with a prominent cholestatic componentprimary sclerosing cholangitis, chronic liver allograft rejection, and extrahepatic biliary atresia. By contrast, in cases of non-cholestatic chronic liver disease, portal lymph nodes were less commonly enlarged and contained little or no lipofuscin pigment. The one exception in which large amounts of pigment were present was a case of "cryptogenic" cirrhosis occurring in a 20 year old woman. Although there were no specific clues to aetiology, the histological pattern was suggestive of chronic biliary disease with large amounts of bile pigment and moderate amounts of copper-associated protein present.

A previous study by Roggendorf described lipofuscin granules in portal lymph nodes in a variety of conditions including alcoholic cirrhosis, sarcoidosis, pancreatic carcinoma and cholelithiasis. ${ }^{8}$ The latter three conditions may all have a cholestatic course.

Lymph node enlargement associated with lipofuscin can also be seen in more distant sites of lymphatic drainage from the liver. Hamazaki described lipofuscin granules in mesenteric lymph nodes from a case of pericholangitis, ${ }^{9}$ and we have seen extensive intra-abdominal lymph node enlargement, associated with pigment deposits in one case of primary biliary cirrhosis and another of extrahepatic biliary atresia at necropsy (unpublished observation).

The duration of disease also seems to be an important factor in determining the amount of pigment present. The largest amounts were seen in cases of primary biliary cirrhosis and primary sclerosing cholangitis which generally have a duration of several years before end-stage disease requiring transplantation is reached. Smaller amounts of pigment were present in cases of extrahepatic biliary atresia and chronic liver allograft rejection in which the median duration of illness in this study had been 11 and three months, respectively. Other processes, including rejection and replacement of donor lymphoid cells with those from the host, may help cause enlargement of donor lymph nodes attached to the grafted liver. ${ }^{111}$ As far as we are aware, however, accumulation of lipofuscin has not been previously described in nodes attached to the liver allograft. No portal lymph nodes were detected in cases of acute liver disease including failed non-rejection liver grafts in which the median survival had been 13 days. Severe cholestasis is a common histological finding in many of these conditions ${ }^{4611-13}$ and the absence of any portal lymph nodes in these cases further supports the suggestion that prolonged cholestasis is required to produce the characteristic lymphadenopathy associated with lipofuscin.

Our study also showed another, apparently distinct, population of ceroid laden macrophages in paracortical and mantle zones of portal lymph nodes. 
Although these were clearly distinguishable from sinus histiocytes laden with lipofuscin by histological examination and long $\mathrm{ZN}$ staining, there was some overlap in the staining reactions with PAS-diastase. This is perhaps not surprising as lipofuscin and ceroid are closely related chemically and the two terms are often used interchangeably. Pearse proposes a unifying concept of lipofuscins in which ceroid is regarded as a typical lipofuscin in the early stages of oxidation. ${ }^{14}$ In the context of liver disease ceroid and lipofuscin are generally regarded as distinct entities. Ceroid refers to brown pigment in phagocytes which is PAS-diastase positive but not necessarily acid fast; lipofuscin, on the other hand, represents granular material in hepatocytes that is acid fast and displays a variable degree of PAS positivity. ${ }^{1516}$ The material seen in sinus histiocytes in our cases thus has staining properties corresponding to lipofuscin.

The clinical importance of these two apparently distinct populations of histiocytes containing different types of "lipofuscins" is uncertain. Although they often occurred in the same nodes, it was impossible to establish a definite relation between the presence or amounts of the two pigments present. Overall, ceroid pigment was present in much smaller quantities than lipofuscin and did not seem to be an important factor in producing lymph node enlargement.

Portal lymph node enlargement was a common finding in livers with primary tumours. The histiological appearances, however, were different from those seen in chronic cholestatic liver disease. Nodes draining neoplastic livers showed a mixed picture of simple sinus histiocytosis and follicular hyperplasia and most cases contained no lipofuscin deposits. The appearances were similar to those described in nodes draining other tumour sites, such as the breast and stomach. ${ }^{17-20}$

How can the apparent relation between chronic cholestatic liver disease and the accumulation of lipofuscin pigment in portal lymph nodes be explained? The relation is unlikely to be a simple chemical one. Bile is a substance with a complex chemical composition which includes a mixture of water, electrolytes, bile salts, bilirubin, phospholipids, cholesterol and several enzymes. ${ }^{21}$ Lipofuscin also has a complex composition and the term is used to describe a group of substances which are heterogeneous in their chemical properties. ${ }^{14}$ Chemical analysis is difficult due to pronounced insolubility, ${ }^{22}$ but the pigments seem to be composed of lipids and phospholipids complexed with protein ${ }^{23}$ and are thought to be derived from lipid precursors by an oxidative process involving free radicals. ${ }^{24} \mathrm{~A}$ possible mechanism might be the transport of bile from liver to regional lymph nodes in the early stages of cholestasis and the subsequent oxidation of lipid constituents of bile to produce lipofuscin. The absence of bile pigments in any of the lymph nodes we studied, however (including may nodes attached to livers with considerable intracellular bile) fails to support this hypothesis.

The presence of enzymes such as non-specific esterase, ${ }^{25}$ acid phosphatase ${ }^{14}$ and a variety of other acid hydrolase ${ }^{26}$ in lipofuscin granules suggests that these granules are lysosomal in origin. Why these should selectively accumulate in chronic cholestatic liver disease is uncertain, but it is interesting to speculate on the possible role of copper accumulation. Granules containing copper-associated protein (CAP) are an extremely common finding in periportal hepatocytes in chronic cholestatic liver disease, particularly cases of primary biliary cirrhosis and primary sclerosing cholangitis. ${ }^{27-30}$ The granules containing CAP also contain lysosomal enzymes ${ }^{31-33}$ and stain positively with a variety of stains for lipofusin. ${ }^{34}$ These granules may therefore be a source of lipofuscin that is released from damaged hepatocytes and carried to portal lymph nodes to produce the characteristic lymphadenopathy seen in association with chronic cholestasis. The finding of black, orcein positive granules in $30 \%$ of the nodes with lipofuscin deposits in sinus histiocytes further supports this hypothesis and suggests that CAP mayo also be carried to portal lymph nodes in long standing cholestasis. The orcein positive granules had different distribution from lipofuscin, however, being mainly present in paracortical/mantle zone histiocytes in association with ceroid pigment.

The functional importance of lipofuscin induced portal lymphadenopathy is uncertain. Most cases are asymptomatic with enlarged nodes only detected at laparotomy or necropsy. Occasional cases of lymph node enlargement sufficiently gross to cause bile duct compression have been described. ${ }^{35}$ They may convey a false impression of neoplastic disease, a problem likely to be encountered by the radiologist with the use of increasingly sensitive imaging techniques as well as the surgeon.

From a diagnostic viewpoint, our study suggests that the finding of large amounts of lipofuscin in enlarged portal lymph nodes is a good indicator of underlying chronic cholestatic liver disease.

We are indebted to clinical colleagues on the liver unit, Queen Elizabeth Hospital, Birmingham, for providing us with the opportunity to study their patients. Mrs J Fitzmaurice provided technical assistance, supported by a grant from the endowment funds of the United Birmingham Hospitals. Miss M Dee typed the manuscript.

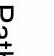




\section{References}

1 Sherlock S. Primary biliary cirrhosis (chronic intrahepatic obstructive jaundice). Gastroenterology 1959;37:574-86.

2 Benbow EW, McMahon RFT. Chromolipid induced portal lymphadenopathy in primary biliary cirrhosis. Histopathology 1987;11:971-7.

3 Wight DGD. Pathology of liver transplantation. In: Calne RY, ed. Liver transplanation: the Cambridge/Kings College experience. 2nd edition. London: Grune and Stratton, 1987:385-435.

4 Hübscher SG, Clements D, Elias E, McMaster P. Biopsy findings in cases of rejection of liver allograft. $J$ Clin Pathol 1985;38:1366-73.

5 Grond J, Gouw ASH, Poppema S, Slooff MJH, Gips GH. Chronic rejection in liver transplants. A histopathologic analysis of failed grafts and antecedent serial biopsies. Transplant Proc 1986;18 (Suppl 4):128-35.

6 Vickers CR, Hübscher SG, Skidmore SJ, McMaster P, Elias E. Sporadic non-A non-B hepatitis as a cause of acute liver failure. In: Viral hepatitis and liver disease. Proceedings of the VIth International Meeting. New York: AR Liss, 1988:561-3.

7 Hübscher SG, Adams DH, Buckels JAC, McMaster P, Neuberger J, Elias E. Massive haemorrhagic necrosis of the liver following liver transplantation. J Clin Pathol 1989;42:360-70.

8 Roggendorf W, Gross U. Ultrastructural and histochemical investigations of spindle-shaped corpuscles in phagocytic cells of lymph nodes. Beitage für Pathologie 1977;161:267-82.

9 Hamazaki Y. Ueber ein neues, säurefeste Substanz führendes Spindelkörperchen der menschlicher Lymphdrüsen. Virchows Arch (Cell Pathol) 1938;301:490-522.

10 Porter KA. Pathology of the orthotopic homograft and heterograft. In: Starzl TE, ed: Experience in hepatic transplantation. Philadelphia: WB Saunders, 1969:422-68.

11 Demetris AJ, Jaffe R, Starzl TE. A review of adult and paediatric post-transplant liver pathology. Pathol Annu 1987;Part 2: 347-86.

12 Wight DGD. Differential diagnosis of cholestasis in liver allografts. Transplant Proc 1986;18(Suppl 4):152-6.

13 Williams JW, Vera S, Peters TG, et al. Cholestatic jaundice after hepatic transplantation. A non-immunologically mediated event. Am J Surg 1986;151:65-70.

14 Pearse AGE. Pigments and pigment precursors. In: Histochemistry, theoretical and applied. Vol 2, 4th Edition. Edinburgh: Churchill Livingstone, 1985:874-928.

15 Poulsen H, Christofferson P. Atlas of liver biopsies. Munksgaard: JB Lippincott Co, 1979:122.

16 Scheuer PJ. Liver biopsy interpretation. 3rd Edition. London: Ballière Tindall, 1978:19-22, 245-9.

17 Hunter R, Ferguson D, Coppleson L. Survival with mammary cancer related to interaction of germinal centre hyperplasia and sinus histocytosis in axillary and internal mammary lymph nodes. Cancer 1975;36:528-39.

18 Symmers W St C. The lymphoreticular system. In: Systemic pathology. 2nd Edition. Edinburgh: Churchill Livingstone, 1978:550-71.
19 Fisher E, Redmond C, Fisher B. Pathologic findings from the National Surgical Adjuvant Breast Project. Cancer 1980;46:908-18.

20 Fisher E, Kotwal N, Herman C, Fisher B. Types of tumour lymphoid response and sinus histiocytosis. Arch Pathol Lab Med 1983;107:222-7.

21 Desmet VJ. Cholestasis. In: Anthony PP, MacSween RNM, eds. Recent advances in histopathology. No 12. Edinburgh: Churchill Livingstone, 1984:146-57.

22 Robbins SL, Cotran R, Kumar V. Pathologic basis of disease. 3rd Edition, Philadelphia: WB Saunders, 1984:23.

23 Tombald RD. Studies on chemical composition of lipofuscin (age pigment) from normal human brain. Lipids 1975;10:383-90.

24 Bancroft JD, Stevens A. The lipofuscins. In: Theory and practice of histological techniques. 2nd Edition. Edinburgh: Churchill Livingstone, 1982:250-2.

25 Gomori G. Histochemistry of human esterases. J Histochem Cytochem 1955;3:479-84.

26 Goldfischer S, Villarverde H, Forschirm R. The demonstration of acid hydrolase, thermostable reduced diphosphopyridine nucleotide tetrazolium reductase and peroxidase activities in human lipofuscin pigment granules. $J$ Histochem Cytochem 1956;14:641-52.

27 Salaspuro M, Sipponen P. Demonstration of an intracellular copper-binding protein by orcein staining in longstanding cholestatic liver diseases. Gut 1976;17:787-90.

28 Jain S, Scheuer PJ, Archer B, Newman SP, Sherlock S. Histological demonstration of copper and copper-associated protein in chronic liver diseases. J Clin Pathol 1978;31:784-90.

29 Nakamura Y, Karino T, Ohta G. Orcein positive granules in the hepatocytes in chronic intrahepatic cholestasis. Virchows Arch (Pathol Anat) 1979;382:21-30.

30 Guarascio P, Yentis F, Cevikbas U, Portmann B, Williams R. Value of copper-associated protein in diagnostic assessment of liver biopsy. J Clin Pathol 1983;36:18-23.

31 Goldfischer S. Liver cell lysosomes in Wilson's disease: acid phosphatase activity by light and electron microscopy. Am J Pathol 1963;43:511-25.

32 Goldfischer S, Moskal J. Electron probe micro analysis of liver in Wilson's disease. Am J Pathol 1966;48:305-15.

33 Goldfischer S, Sternlieb I. Changes in the distribution of hepatic copper in relation to the progression of Wilson's disease (hepatolenticular degeneration). Am J Pathol 1968;53:883-907.

34 Goldfischer S, Popper H, Sternlieb I. The significance of variations in the distribution of copper in liver disease. Am J Pathol 1980;99:715-30.

35 Summerfield JA, Elias E, Hungerford GD, Nikapota VLB, Dick $R$, Sherlock $S$. The biliary system in primary biliary cirrhosis. A study by endoscopic retrograde cholangiopancreatography. Gastroenterology 1976;70:240-3.

Requests for reprints to: Dr S G Hübscher, Department of Pathology, The Medical School, University of Birmingham, Birmingham B15 2TJ, England. 